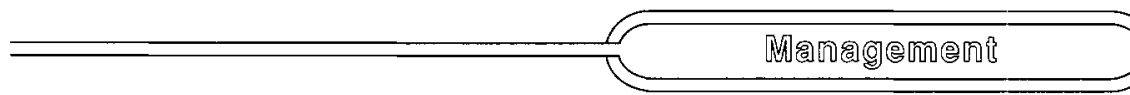

\title{
Designing and Delivering Effective Presentations
}

\section{Public speaking becomes less stressful and more effective if the preparation and delivery are carried out in a step-by-step manner. Here's a guide for oral-presentation planning}

\section{by Judi Brownell}

SUCCESSFUL LEADERSHIP requires that you present your ideas clearly and concisely. Regardless of how well you know your particular area of expertise, it takes a skilled communicator to motivate employees, implement action plans, and build a successful team. This article provides an outline of the strategies and principles of effective presentational speaking.

A good place to start is by learning the following points. This set of concepts, which I call MAPP, can be your guide to presentational speaking by keeping you focused on the four keys to effectiveness.
M otivation: Recognize the importance of effective speaking to your personal and professional development. Accurately access your personal strengths and weaknesses as a speaker, and work hard to improve your speaking skills.

A ttitude: Think positive! Public speaking allows you to be creative and in complete control of the speech situation.

$\mathbf{P}$ rinciples: Understand the basic principles of presentational speaking and apply them regularly to your job.

$\mathbf{P}$ ractice: Seek opportunities to practice your speaking skills.

By following this MAPP and the guidelines that follow, you will be well on your way to delivering powerful presentations. The guidelines revolve around three main steps: (1) Analyze the situation requiring the presentation;

(2) Prepare to speak; and

(3) Deliver the presentation.

\section{Analyze the Communication Situation}

Before you determine what you want to say, consider your audience and the reason for your presentation. The information you present must be "the right stuff,"

Judi Brownell, Ph.D., is an associate professor of communication at the Cornell University School of Hotel Administration. 
selected on the basis of your listeners and the specifics of the occasion. Unless you accurately analyze your listeners' needs and expectations, the chances of accomplishing your goals are slim.

Listen before you speak. Your speaking will be only as good as your ability to listen ahead of time. Effective speakers learn to (1) listen objectively to all of the facts before making any judgment or evaluation, and (2) observe non-verbal cues to understand people's ideas and feelings more accurately.

Listening objectively involves letting go of the common tendency to become emotionally attached to those topics that are important to us. When we personalize issues at an emotional level, we become defensive when others disagree or find fault with our ideas. The result is that we can't be objective in assessing all sides of an issue.

As for nonverbal cues, pay careful attention to your prospective listeners' tone of voice and observe their facial expressions and body language for indications of their attitudes and opinions.

Understand the speaking occasion. Find out as much as possible about the events that surround your particular presentation and the speaking environment itself. Factors that influence your listeners' responsiveness include:

- The type, quality, and length of presentations that immediately precede your speech,

- The time of day,

- The room arrangement and where key individuals will be sitting,

- The availability of audiovisual equipment,

- Whether attendance at your presentation is voluntary or mandatory, and

- The degree to which participants have prepared for the event.

\section{Prepare to Speak}

Several steps will help you prepare for the speaking event: (1) develop a clear statement of purpose, (2) research your presentation, (3) organize your facts in some logical sequence, (4) develop the main points of your speech, and (5) create an effective introduction and conclusion.

Clarify your purpose. The goal of your presentation will influence the information you select to share, the particular emphasis you place on various facts, and the way you arrange other material that's available. A statement of purpose will keep you on track and help ensure that your stated goal doesn't get derailed by personal and political motivations (e.g., the desire to "look good" in front of your colleagues, or to point a finger of blame at someone).

Collect and select information. Research your presentation to verify the facts being presented and to uncover new and relevant material.

Organize and develop your facts. The more important the occasion, the greater the amount of information required to do a really good job; the greater the amount of raw data to be sorted, the more difficult it is to get started. Here's a way to organize and develop the materials you have gathered.

Formulate a thesis. The specific way in which you select and develop your points will influence the way in which your ideas are perceived. It's important, then, to formulate a statement of your position on the issue being addressed. That will be your thesis. The thesis is an opportunity to lead the listeners to your vision of what the desired outcome should be and to persuade them to agree with your point of view (or, at least, to get them to consider it carefully).

Determine the pattern of organization. The first step to effective organization is categorization. There are a variety of ways to sort ideas on any given topic, and each method of sorting suggests a different pattern of organization. The five most common are topical, spatial, chronological, enumerative, and problem-solving, as defined below.

- Topical: Organization by specific problems or questions

- Spatial: Organization by areas (e.g., food and beverage, rooms, front desk)

- Chronological: Organization by time, as in a sequence of events leading up to a current situation

- Enumerative: Organization by the relevance, significance, or complexity of the information

- Problem-solving: Organization by presenting the problem(s) and recommending a solution (or solutions)

The way you sort your information affects the emphasis each idea receives and ultimately the way the information will be interpreted by others. Clear patterns of organization will ensure that your listeners understand what you have to say. This order or pattern of organization also makes it easier for you to remember the sequence of your ideas.

Develop your main points. When speaking, less really is more. The goal is to provide vivid, meaningful support for the ideas you want to get across rather than to overwhelm your listeners with too many details.

A good way to test the "listenability" quotient of your presentation is to see how many different types of verbal "development" you have used to clarify each of your main points, as explained below. There are at least eight methods of development that you can use: example, literal comparison, figurative comparison, definition, quotation, testimony, statistic, and humor. 
Example. Make your point by citing a real-life example of the situation or problem, complete with the level of detail necessary to prove its accuracy.

Literal comparison. Compare your situation with the corresponding circumstances at another organization.

Figurative comparison. Draw on a story or concept, real or made up, that conjures up the image you want to convey.

Definition. Provide a dictionary definition of your topic.

Quotation. Use a quotation from someone your listeners know that is relevant to the topic.

Testimony. Provide the insights of experts in the field or subject area.

Statistic. Use numbers and figures to make your points and comparisons.

Humor. Incorporate anecdotes, jokes, and silly sayings into your presentation when doing so is appropriate.

The body of your presentation, then, should consist of approximately three or four main points, each thoroughly developed using the methods just described. You could conceivably use all eight types of development listed above to make a single point, but it's more likely that, for time's sake, you'll depend on three or four carefully chosen techniques to clarify and emphasize each main idea.

You'll want to focus on establishing the relationships between the points you're making, and on how those points relate to the purpose (thesis) of your presentation. Linking one main point to the next and each point back to your thesis isn't always easy, but it will keep both you and your listeners on track.

Create an introduction and conclusion. Your next step is to create an effective introduction and conclusion, as these will have a significant impact on the effectiveness of your presentation.

\section{An Outline for Effective Presentational Speaking}

\section{Analyze the Communication Situation}

A. Listen before you speak

1. Listen objectively

2. Consider nonverbal cues

B. Understand the speaking occasion

1. Do other presentations precede yours?

2. Time of day

3. Where your presentation falls on the agenda

4. Room arrangement, including where key people will sit

5. Whether audiovisual equipment is required

6. Degree to which participants have prepared for the event

\section{Prepare to Speak}

A. Clarify your purpose

B. Collect and select information

C. Organize and develop ideas

1. Formulate a thesis

2. Determine the pattern of organization for your material

a. Topical

b. Spatial

c. Chronological

d. Enumerative

e. Problem-solving

D. Develop your main points

1. Example

2. Literal comparison

3. Figurative comparison

4. Definition

5. Quotation

6. Testimony

7. Statistics

8. Humor

E. Create an introduction and conclusion

1. Introduction
a. Attention material
b. Orientation
c. Relate the topic to your audience

2. Conclusion
a. Summary
b. Recall some point from your introduction or thesis
c. Restate the implications for the listeners
d. Provide a strong image on which to end

\section{Deliver the Presentation}
A. Style of delivery
B. Overcome nervousness
C. Use appropriate nonverbal communication
1. Voice
2. Eye contact
3. Body movement and gesture
4. Visual aids
D. Control the nonverbal environment
E. Respond to questions

Introduction. Every introduction must accomplish three goals. First, it must gain the listeners' attention. Next, it must clearly present the speaker's purpose and outline the main points that will be covered. Finally, it must relate the topic to the listeners' perspectives and give the audience a good reason for listening.

To gain the listeners' attention, you might employ one of several attention-getting strategies:

- Begin with a startling statement,

- Begin with a rhetorical question,

- Describe an actual or hypothetical situation or event,

- Use a relevant quotation, 
- Refer to an event or experience well-known to the entire audience, or

- Demonstrate the item or process you will be discussing.

To present your purpose for speaking, use some form of the thesis that you already developed. Remember, your thesis is the overall position you're taking or the point that you're making with regard to your topic. It's also important to give your listeners a mental picture of what is to come by "blueprinting" the main points you plan to cover. Such an overview helps them to think about your topic. Not only will their listening improve, but their memory will as well.

To relate your topic to your listeners' perspectives and to provide a reason for them to listen, the best approach is to assume that each member of your audience has been invited somewhere special. Each is anxious to leave, and may do so at any moment. Your job is to convince your listeners that it is vital for them to stay and hear what you have to say. By focusing on the needs of your audience in this way, you'll automatically be sensitive to their point of view and to their degree of involvement with the topic.

Conclusion. A well-planned conclusion can turn a mediocre presentation into one that is memorable. Here, the first step is to avoid the conclusion TRAP:

$T$ railing off vocally at the end instead of maintaining adequate volume and energy;

$\mathbf{R}$ ushing through the end of the presentation, perhaps because time is running out;

A sking for questions at the end of your presentation instead of providing a strong concluding statement (you should ask for questions during your speech); and

$\mathbf{P}$ retending at the end that you don't care what your listeners think.
Another point to keep in mind is that listeners generally retain only about 20 percent of the total message. That's why the first rule of presentational speaking is so important: Tell them what you're going to tell them, tell them, then tell them what you've told them.

Ideas can be repeated and reinforced in a variety of ways. Although a summary is often necessary, creative and thoughtprovoking approaches will add interest to your speech. For example, refer back to some aspect of the material you used to grab their attention, or to your introduction. Because you want to leave your listeners with a vivid impression of how the topic affects them personally, do not replace a good, solid conclusion with a call for questions.

\section{Deliver Your Presentation}

A well-prepared presentation is much easier to deliver than one that is put together in a hurried, haphazard manner. This is important to note because the actual delivery has a tremendous influence on your listeners' reactions. To do your best in front of a group, you need to (1) choose the appropriate style of delivery, (2) overcome your nervousness, (3) use appropriate nonverbal communication, and (4) control the environment.

Style of delivery. All along we've been discussing an extemporaneous style of presentation. That means the speech is well prepared, thoroughly researched, carefully organized, and thoughtfully developed. Minimal notes (usually note cards) are used for the main points, statistics, quotations, and other selected material. The sequence of ideas is memorized, but the words used to express those ideas are not.

Alternative styles of delivery include those that involve com- plete memorization of a script, the reading word-for-word of prepared text, or the impromptu presentation that is neither planned nor practiced. While impromptu presentations are sometimes unavoidable, I urge you never to make a presentation by reading or memorizing a fully prepared script.

Overcome your nervousness. Ninety-nine percent of speech anxiety stems from one or more of the following problems: focusing on yourself rather than on your presentation, poor preparation, and lack of genuine personal interest in the topic. Here are some techniques for overcoming speech anxiety:

- Breathe deeply before getting up to speak;

- Get enough rest prior to the presentation;

- Look at your audience for a few seconds before you begin to speak;

- Pretend someone in the audience just asked a question about your topic and you're about to answer it;

- Find a friendly face to look at until you feel more comfortable;

- Concentrate on your topic, rather than on how you may appear to your listeners; and

- If your hands shake, avoid doing things that may show your shakiness to the audience-such as trying to write on an overhead transparency.

When you're ready to start, don't rush into your introduction. Look out over the audience and make eye contact with your listeners. Plan to talk to them informally; then, as you speak, keep asking yourself: "Do they understand what I've said? Have I been clear?"

The secret here is to not focus on your first, most-natural thought: "How am I doing?" Instead, concentrate on the real, most-important question: "Am I accomplishing my purpose?" 


\section{Appropriate nonverbal} communication. Your audience will respond to three different types of nonverbal communication: your voice, your body movements and gestures, and your eye contact. You can become a more credible and effective speaker simply by learning how nonverbal behavior affects perceptions of your knowledge and sincerity.

Voice. You can project confidence by manipulating three characteristics of your voice: pitch, volume, and rate of speech. An unnaturally high pitch may belie a speaker's apparent composure, while the appropriate (usually instinctive) use of inflections can be very effective in driving home important points. Volume involves a consistently strong sound at a level appropriate for the environment. And rate of speech is merely a matter of not speaking too fast or too slowly, but of maintaining a quick pace characterized by attention-getting variation.

Body movements and gestures. Most nonverbal behaviors have different meanings to different ethnic and cultural groups. This is important to learn because movements, gestures, and other nonverbal behaviors can communicate powerful messages regarding status, power, gender differences, and interpersonal attraction. Keep in mind that what I say here is an American view of nonverbal communication.

You want your nonverbal communication to reinforce, not contradict, the message you're trying to convey. A moderate amount of gesture and movement can help hold the listeners' attention, but any such activity must appear purposeful and not a result of nervous energy. Movement that is deliberate contributes to your credibility while fidgeting and unusual mannerisms communicate anxiety and reduce your credibility. Basically, you want to avoid behaviors that focus attention on what you're doing rather than on what you're saying.

Eye contact. Strong eye contact communicates interest and personalizes the exchange. For example, you know how important eye contact can be in the service encounter. While the norms dictating eye contact are culturally determined, within those accepted restrictions it's always possible to show your acceptance of the other person as a communication partner through eye contact.

One of the easiest ways to ensure eye contact with an audience is mentally to divide the group into three sections. Begin by looking at the center section, then move your focus to one of the sides and then to the other. Choose a person (or two) from each of the sections and really make eye contact with her or him. If you've heard that you can look just above your listeners' heads and not be noticed doing so, don't believe it! Besides, there's no substitute for the connection that is made through direct eye contact.

Control the environment. Many external elements contribute to the quality of human communication. The aspects that come immediately to mind are seating arrangements, furniture and decor (including colors), position from which the speaker is expected to speak, placement of visuals, the temperature of the room, and the time of day.

Although many of these factors cannot be changed, some can be deliberately planned or arranged to achieve maximum control over the speaking environment.

\section{Responding to Questions}

Fear of not knowing the answer to a question ranks at the top of the list when business speakers talk about their worst nightmares. If you're among those who dread questions from the audience, here's one way to try to overcome that anxiety.

First, try to put your expertise into perspective. Assuming you've done your homework and you're well prepared for the presentation, the audience will acknowledge that it's impossible for one person to know absolutely everything about a topic.

Next, remember that you're in control of the discussion and that you have options. Depending on the specifics of the situationincluding the apparent motive of the questioner-you can answer the question, deflect the question, or defer the question to a later time (e.g., the end of the session or a private meeting).

Try to anticipate the questions that might be asked, and practice responding to those questions by having a colleague listen to your presentation. Above all, do not become defensive. Listen carefully, and make certain you understand each question. If you don't know an answer, admit it. You may choose to speculate or to offer an opinion in response to a question, but be sure to qualify your answer so the audience knows you're not pretending to know the absolute truth about that particular question.

Don't allow one person to dominate the time allowed for questions, and try to avoid situations involving an extended argument of a point that is not of major interest to the entire group.

Finally, remain positive and professional. Your attitude and demeanor throughout the presentation will go a long way toward defining the image of you that your listeners will take with them when they leave the room. $\square$ 